\title{
ORIGINAL RESEARCH: TRADITIONAL CHINESE MEDICINE Protective effect of ganoderan on renal damage in rats with chronic glomerulonephritis
}

\author{
Wei-De Zhong1, 2 \\ Hui-Chan $\mathrm{He}^{1}$ \\ Ru-Biao Ou ${ }^{1}$ \\ Xue-Cheng $\mathrm{Bi}^{2}$ \\ Qi-Shan Dai ${ }^{1}$ \\ Zhao-Dong Han ${ }^{1}$ \\ Yu-Xiang Liang ${ }^{1}$ \\ Yong-Kan Ye ${ }^{1}$ \\ Wei-Jun Qin ${ }^{3}$ \\ $\mathrm{Ze} \mathrm{\mathbf {Li } ^ { 4 }}$ \\ Guo-hua Zeng 4 \\ Gang Zhu ${ }^{5}$
}

Corresponding authors are Weide Zhong and Kan Gong. The first two authors contributed equally to this article.
${ }^{1}$ Guangzhou First Municipal People's Hospital, Affiliated Guangzhou Medical College, Guangzhou 510180, China

${ }^{2}$ Nanfang Medical University Guangzhou 510180,

${ }^{3}$ Beijing Hospital of Ministry of Public Health, Beijing, China

${ }^{4}$ XiJing Hosptial,The Fourth Military Medical University, Xian China

${ }^{5}$ Department of Urology, Peking University first Hospital, Beijing 100034

Manuscript submitted 16th March, 2008 Manuscript accepted 28th June, 2008

Clin Invest Med 2008; 31 (4): E212-E217.

\begin{abstract}
Purpose: To investigate the protective effect of ganoderan on renal damage in rat models with chronic glomerulonephritis induced by adriamycin.

Methods: 48 healthy Sprague-Dawley rats were randomly divided into three groups: control, nephritic model and ganoderan treatment groups. Changes of the following indices in the three groups were observed 6 weeks after treatment: 24-hour urine protein, albumen, serum creatinine, cholesterol. Histopathological observations of the renal cortex were made by light and electron microscopy.

Results:_Compared with controls, levels of 24-hour urine protein $(9.60 \pm 0.57 \mathrm{mg} / \mathrm{d} v s .82 .50 \pm 3.18 \mathrm{mg} / \mathrm{d})$, serum creatinine $(35.25 \pm 2.63 \mu \mathrm{mol} / \mathrm{L}$ vs. $44.75 \pm 8.06 \mu \mathrm{mol} / \mathrm{L})$ and cholesterol $(1.15 \pm 0.10 \mathrm{mmol} / \mathrm{L}$ vs. $4.02 \pm 0.25 \mathrm{mmol} / \mathrm{L})$ of rats in the nephritic model group were increased $(P<0.05)$, and the concentration of albumen was decreased $(35.98 \pm 1.34 \mathrm{~g} / \mathrm{L}$ vs. $19.05 \pm 0.62 \mathrm{~g} / \mathrm{L}, P<0.05)$. Ganoderan administration decreased 24-hour urine protein $(82.50 \pm 3.18 \mathrm{mg} / \mathrm{d} v s$. $45.01 \pm 3.94 \mathrm{mg} / \mathrm{d}, P<0.05)$. Following ganoderan, the
\end{abstract}

pathological changes in kidney tissue were improved compared with those in the nephritic model group.

Conclusion: Ganoderan exerts protective effects in rats with chronic glomerulonephritis induced by ADR. Ganoderan reduced 24-hour urine protein, serum creatinine, cholesterol, improving renal function and reducing the severity of renal injury.

Ganoderan contains the active components of Ganoderma lucidum (Ganoderma or lingzhi) ${ }^{1}$, an Asian medicinal mushroom that has been used for two thousand years for the treatment of various diseases such as hypertension ${ }^{2}$, hyperlipidemia ${ }^{3}$, diabetes ${ }^{4}$, hepatitis ${ }^{5}$, allergy ${ }^{6}$, anti-aging, anti-fibrosis ${ }^{7}$ and cancer ${ }^{8}$. Recent studies have indicated that the extract of Ganoderma lucidum-ganoderan, also has a wide range of pharmacological actions including suppressing inflammation and scavenging free radicals. ${ }^{9}$ 
Adriamycin (ADR) causes chronic nephropathy in rats, which corresponds clinically and histologically to chronic glomerulonephritis, focal and segmental glomerulosclerosis. ${ }^{7,} 8$ In the present study, we prepared rat models with chronic glomerulonephritis by the intravenous injection of ADR. Rats in the ganoderan treatment group were fed ganoderan for 7 days. The following indices were observed after 6 weeks treatment: urine protein quantitation (UPr) of $24 \mathrm{~h}$, serum cholesterol (Ch), serum albumin (Alb), serum creatinine $(\mathrm{sCr})$. Histopathological examination of rat renal cortex was made by light and electron microscopy. Changes in these markers were compared among the control, nephritic model, and ganoderan treatment groups to investigate the protective effects of ganoderan on rats with chronic glomerulonephritis.

\section{Materials and Methods}

\section{Animals}

Forty-eight healthy Sprague-Dawley (SD) rats of sanitation grade, 5-6 weeks old and approximately $200 \pm 25 \mathrm{~g}$, were purchased from the Department of Laboratory Animal Science, Guangzhou Medical College (Guangzhou, China) respectively. The rats were housed and given a commercial diet and tap water for 1 week before experimentation at $24^{\circ} \pm 1^{\circ} \mathrm{C}$ and $50-70 \%$ humidity under a $12 \mathrm{~h}$ light-dark cycle. Experiments were performed according to the "Guidelines for the Care and Use of Experimental Animals" of the Chinese Association for Laboratory Animals. After one-week adaptation, rats were randomly divided into three groups: control, nephritic model and ganoderan treatment groups with 16 rats in each group.

\section{Materials}

Adriamycin (ADR, " 02D020-23214-92-8) was purchased from Asia Talent Chemical Supplier. Ganoderan, the active ingredient of the capsule containing mythic fungus spore powder, $0.3 \mathrm{~g} /$ capsule, was pur- chased from the Fuzhou Institute of Green Valley BioPharm Technology (Fuzhou, China). 10 mg ADR was added to physiological saline to make a solution with a concentration of $1 \mathrm{~g} / \mathrm{L}$. The contents of the capsule containing ganoderan were dissolved in distilled water to provide $2 \mathrm{ml} / \mathrm{rat}$.

\section{Preparation of Animal Models and Drug Treatment}

In the nephritic model and the ganoderan treatment groups, chronic glomerulonephritis was induced with ADR, $3.5 \mathrm{mg} / \mathrm{kg}$, injected into the tail vein of conscious rats twice, 7 days apart. Rats in the control group received the same volume of physiological saline. Rats in the ganoderan group were given mythic fungus spore powder, $5 \mathrm{~g} / \mathrm{kg}$, intragastrically for 7 days. Rats in the control and nephritic groups were given the same amount of distilled water. During the experiment, all rats were maintained in a standard environment with $12 \mathrm{~h}$ daytime, $12 \mathrm{~h}$ night, temperature $24^{\circ} \pm 1^{\circ} \mathrm{C}$ and humidity $50 \%-70 \%$. Animals fed freely on whole-value particle animal feedstuff, made by our animal experimental centre, and tap water.

\section{Urine Protein and Blood Biochemical Detection}

24h UPr (expressed as urinary protein in $\mathrm{mg} / \mathrm{ml}$ glomerular filtrate in 24h) was determined using spectrophotometry, after 3\% sulfosalicylic acid precipitation of urine which was collected from rats individually housed in metabolic cages for $24 \mathrm{~h}$ before ADR administration and $6 \mathrm{wk}$ after drug treatment. Simultaneously, blood samples_were drawn from rats in the three groups. Biochemical parameters ( $\mathrm{sCh}, \mathrm{Alb}, \mathrm{sCr}$ ) were measured by standard assays using diagnostic kits (Roche AG, Switzerland) and the biochemical analyzer Cobas Mira plus (Roche AG, Switzerland)

\section{Histopathological Observation}

Six weeks after drug treatment, rats were anaesthetized with $10 \%$ chloral hydrate, $0.5 \mathrm{ml} / 100 \mathrm{~g}$, by i.p.injection. One kidney was removed from each rat 
TABLE 1. Comparison of the mouse biochemical indicators in different groups, 49 days after $\operatorname{drug}$ treatment $(\bar{X} \pm s)$

\begin{tabular}{l|c|c|c|c|c}
\hline Groups & Cases & $24 \mathrm{~h} \operatorname{UPr}(\mathrm{mg} / \mathrm{d})$ & $\mathrm{Alb}(\mathrm{g} / \mathrm{L})$ & $\mathrm{sCr}(\mu \mathrm{mol} / \mathrm{L})$ & $\mathrm{Ch}(\mathrm{mmol} / \mathrm{L})$ \\
\hline Control & 16 & $9.60 \pm 0.57$ & $35.98 \pm 1.34$ & $35.25 \pm 2.63$ & $1.15 \pm 0.10$ \\
\hline adriamycin group & 16 & $82.50 \pm 3.18^{*}$ & $19.05 \pm 0.62^{*}$ & $44.75 \pm 8.06^{*}$ & $4.02 \pm 0.25^{*}$ \\
\hline Ganoderan group & 16 & $45.01 \pm 3.94^{*}$ & $24.48 \pm 0.91^{*}$ & $45.25 \pm 5.68^{*}$ & $2.61 \pm 0.29 * \#$ \\
\hline
\end{tabular}

${ }^{*} P<0.05$, comparison with the control group;

${ }^{\#} P<0.05$, comparison with the adriamycin-induced nephritic model group.

and fixed in $10 \%$ formalin, embedded in paraffin and examined in multiple consequent sections. The histopathological study was carried out using HE staining (hematoxylin -eosin) and observed by light microscope. In addition, other partial kidney tissues were fixed with 3\% glutaraldehyde and $1 \%$ osmic acid, and stained with uranyl acetate and citric acid. Histopathological changes in the rat renal cortex were also observed by electron microscopy.

\section{Statistical Analysis}

Data are presented as a mean \pm standard deviation ( $\bar{X} \pm s$ ) obtained from three independent experiments. Statistical analysis was determined by Dunnett $t$ test (2-sided). $P$ values $<0.05$ were considered statistically significant.

\section{Results}

\section{Effect of Ganoderan on the changes of biochemical indicator in rat models}

The biochemical indicators in three groups are shown in Table 1.

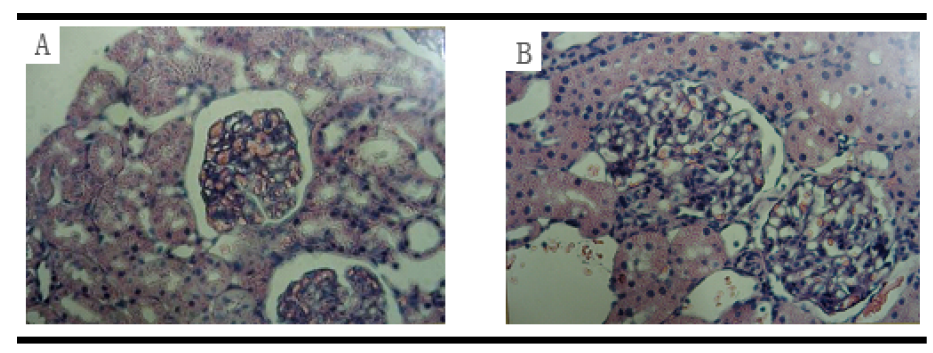

FIGURE 1. Renal pathological changes in rats of control group observed by light microscopy.
Albumen levels in the nephritic group were lower than in the control $(P<0.05)$, but $\mathrm{sCr}$ and $\mathrm{Ch}$ were increased $(P<0.05)$, demonstrating the successful production of the nephritic model. Two_weeks after ADR, hyperproteinuria had occurred in the nephritic model rats and continued to increase. Six weeks after ADR, it was higher than in the control group $(P<0.05)$. After ganoderan administration, urine protein and Ch levels were lower than those in the nephritic model group.

\section{Effect of Ganoderan on renal pathology}

\section{Light microscopy}

Renal tissues in the control group served as negative controls (Figure 1).

In the nephritic group, glomerular mesangium regions were expanded, intercapillary cells increased and basal material multiplied. The lumen of the blood vessels was narrowed and even disappeared. A few renal glomeruli and capsules were partly adherent. There were some casts in the renal interstitium with increased infiltration of inflammatory cell in the renal glomeruli and interstitium (Figure 2).

In the ganoderan treatment group, hyperplasia in the glomerular mesangium regions was reduced,

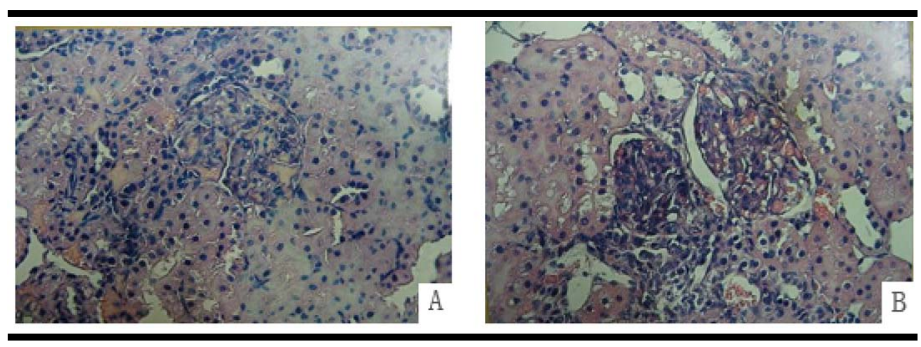

FIGURE 2. Renal pathological changes in rats of nephritic model group observed by light microscopy. 


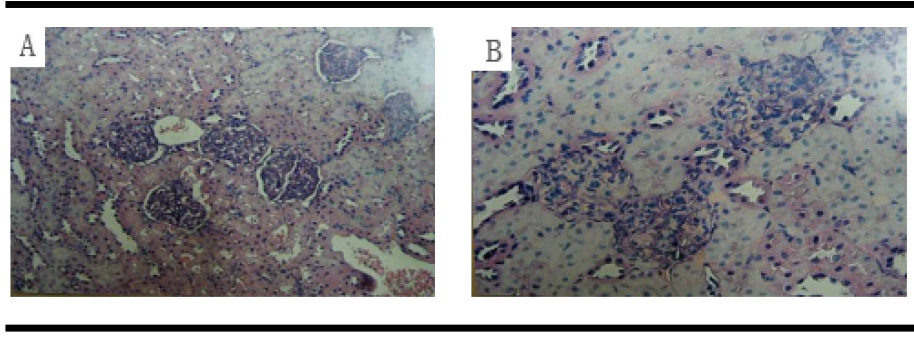

FIGURE 3. Renal pathological changes in rats of ganoderan treatment group observed by light microscopy.

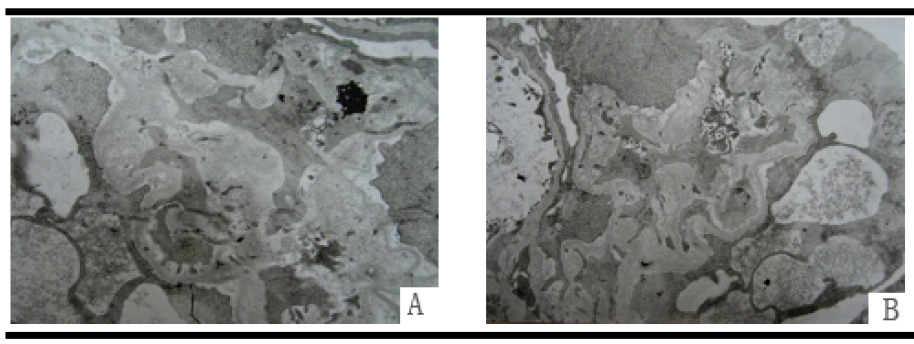

FIGURE 5. Renal pathological changes in rats of nephritic model group observed by electron microscopy.

pathological changes in the basal membrane were not obvious and there was no adherence in the renal capsules. Pathological changes in the renal tubules and interstitium were also improved with fewer casts. There was some infiltration of inflammatory cells in the renal glomeruli and interstitium, but less than in the nephritic model (Figure 3).

\section{Electron microscopy}

The normal renal tissues served as negative control for electron microscopy (Figure 4).

In the nephritic group, mesangial regions were expanded, intercapillary cells propagated, and the foot processes of the foot cells were mixed together and even disappeared with adherence in the renal capsules (Figure 5).

In the ganoderan group, foot processes were partly confluent compared with the nephritic group and, in some regions, the foot processes had recovered completely or were close to normal. Mesangial regions remained mildly expanded with relatively mild hyperplasia in the intercapillary cells (Figure 6).

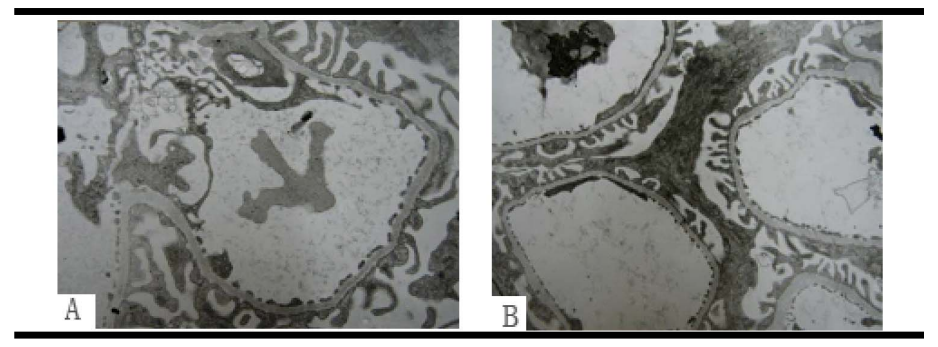

FIGURE 4. Renal pathological changes in rats of control group observed by electron microscopy.

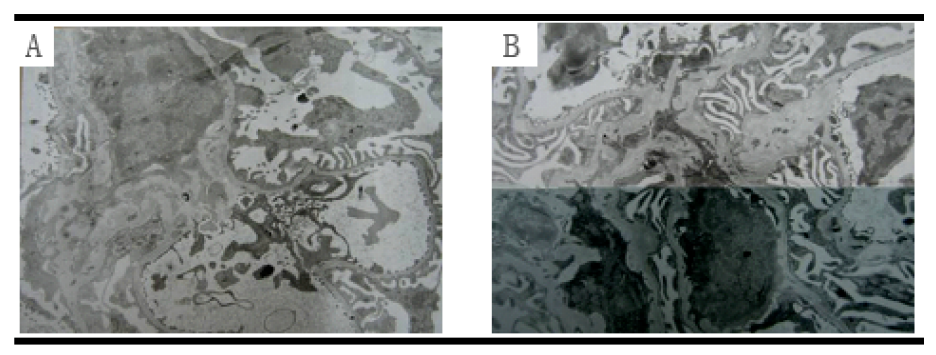

FIGURE 6. Renal pathological changes in rats of ganoderan treatment group observed by electron microscopy.

\section{Discussion}

The fungus Ganoderma lucidum, also known as 'Lingzhi' in Chinese, 'Reishi' in Japanese, and 'Youngzhi' in Korean, is a member of the genus Ganoderma and has been used, traditionally, as a popular herbal medicine for the promotion of health in the Orient. In 1881, the genus Ganoderma was established in the west by the Finnish botanist, Karsten. ${ }^{10}$ Since then, more than 120 species have been reported world-wide. In the present study, the protective effect of ganoderan, the active ingredient of Ganoderma lucidum, on chronic glomerulonephritis was analyzed using the rat models induced by ADR. After ADR administration, 24UPr, $\mathrm{sCh}$ and $\mathrm{sCr}$ levels were increased. Proteinuria has a direct effect on cellular infiltration ${ }^{11}$ and protein overload has been suggested to induce functional alterations of tubular cells, overexpressing proinflammatory mediators. Thus, tubulointerstitial inflammation in rat models with chronic glomerulonephritis corresponded in time with the overt proteinuria as our results. In the ganoderan treatment group, levels of these makers were decreased and renal function was improved. Moreover, 
light microscopical and electron microscopical observation in the nephritic group demonstrated tubulointerstitial changes with cellular infiltration and vacuolar degeneration. Interstitial inflammation has been considered an important determinant of the outcome of glomerular inflammation. Several studies have suggested that myofibroblasts of the interstitium may play a crucial role in the pathogenesis of fibrosis in glomerular diseases. ${ }^{12-14}$ Thus, the tubulointerstitial cellular response and vacuolar degeneration in our rat models with chronic glomerulonephritis emphasize the severity of the illness. On the other hand, attenuation of interstitial inflammation and other renal injury may be secondary to inhibition of proteinuria by the ganoderan medication. These results confirm that ganoderan can postpone the progression of chronic glomerulonephritis.

Many biological effects of ganoderan have been reported. Renal interstitial fibrosis usually shows initial infiltration of lymph cells. Lymph cell culture fluid contains factors that may promote fibrocyte multiplication and collagen synthesis. Ganoderan may inhibit delayed anaphylaxis, restrain the primary power of antibody response and decrease the level of circulating antibody in vivo of rats. ${ }^{11}$ Experiments in vitro have also shown that ganoderan may inhibit the proliferative response of the splenic lymphocytes of rats and the proliferative response of human tonsilla lymphcytes, which suggests that ganoderan hamay have a similar inhibiting effect on both human and rats' lymph cells. Some reports have indicated that, in mice, ganoderan decreases production of IL-2 by the splenic cells (IL-2 can promote the proliferation of T lymph cells ${ }^{12}$ ), and inhibit the mixed lymphocyte culture reaction of the allotype splenic cells. Ganoderan may also increase the peritoneal macrophage ACP, $\beta$ glucuronidase activity and the content of hydrogen dioxide, and antagonize the inhibiting effect of the splenic DNA synthesis by glucocorticoid.$^{13}$ Thus, one may conclude that ganoderan inhibits both the body's cell immunity and humoral immune function.
The mechanism of the protective effects of ganoderan on ADR induced chronic glomerulonephritis is unclear. ADR exhibits its cytotoxic effect mainly by inhibiting topoisomerase II, which causes DNA strand breaks. ADR also may induce ROS production, causing oxidative stress. ${ }^{15}$ The effects of ganoderan on ADR may, at least in part, be based on: (1) attenuation of ADR-induced oxidative stress by ganoderan and (2) inhibition of cells to ADR -induced DNA strand breaks by ganoderan through simulation of the DNA excision repair system.

In conclusion, ganoderan exerts protective effects on ADR-induced chronic glomerulonephritis in rats. By reducing excretory quantity of proteinuria, 24UPr, and $\mathrm{Ch}$, improving renal function and lessening the severity degree of glomerulosclerosis ganoderan retards development of the disease. Further research is needed to confirm the mechanism of the interaction between ganoderan and ADR.

\section{Acknowledgments}

Supported by grants from the Natural Science Foundation of Guangdong Province (No.04003650) and the Key Programs of Science and Technology of Guangzhou city (No. 200323-E4053) and National High Technology Research and Development Project of China (No.2006AA02A245).

\section{References}

1. Xin Wang, Xuan Zhao, Dan Li, Ya-Qing Lou, Zhi-Bin Lin, and Guo-Liang Zhan.: Effects of Ganoderma lucidum Polysaccharide on CYP2E1, CYP1A2 and CYP3A Activities in BCG-Immune Hepatic Injury in Rats. Biol. Pharm. Bull 2007;30:1702-6

2. Thyagarajan A, Jiang J, Hopf A, Adamec J, Sliva D: Inhibition of oxidative stress-induced invasiveness of cancer cells by Ganoderma lucidum is mediated through the suppression of interleukin-8 secretion. Int $\mathbf{J}$ Mol Med 2006;18:657-64.

3. Cheung WM, Hui WS, Chu PW, Chiu SW, Ip NY: Ganoderma extract activates MAP kinases and induces the neuronal differentiation of rat pheochromocytoma PC1 2 cells. FEBS Lett 2000;486:291-6 
4. Lee JM, Kwon H, Jeong H: Inhibition of lipid peroxidation and oxidative DNA damage by Ganoderma lucidum. Phytotherapy Res 2001;15:245-9

5. Yuji Nonaka, Hiroshi Shibata, Masaaki NakaI, Hiroshi Kurihara: Anti-tumor activities of the antlered form of Ganoderma lucidum in allogeneic and syngeneic tumor-bearing rats. Biosci biotechnol biochem 2006;70:2028-34,

6. Lai KN, Chan LY, Tang SC, Leung JC: Ganoderma extract prevents albumin-induced oxidative damage and chemokines synthesis in cultured human proximal tubular epithelial cells.Nephrol Dial Transplant 2006;21:1188-97.

7. Punithavathi D, VenkatesanN, Babu M: Protective effects of curcumin against aminodarone- induced pulmononary fibrosis in rats $\mathrm{Br} \mathrm{J}$ Pharmacol 2003;139:1342-50

8. Lai NS, Lin RH, Lai RS, Kun UC, Leu SC: Prevention of autoantibody formation and prolonged survival in New Zealand Black/New Zealand White F1 rats with an ancient Chinese herb, Ganderma tsugae. Lupus 2000;10:461-5

9. Futrakul N, Boonyen M, Patumraj S, Siriviriyakul P, Tosukhowong P, Futrakul P: Treatment of glomerular endothelial dysfunction in steroid-resistant nephrosis with Ganoderma lucidum, vitamins C, E and vasodilators.Clin Hemorheol Microcirc 2003;29:205-10.

10. Wang G, Zhang J, Mizuno T: Antitumor active polysaccharides from the Chinese mushroom Songshan lingzhi, the fruiting body of Ganoderma tsugae. Biosc Biotec Biochem 1993;57:894-900

11. Ji-Chao Ma, Wan-Xing Cai, Ke-Fu Chen: Treatment of rats' neurospongioma with interferon and interleukin-2. Chinese Journal of Experiment Surgery 1994;11:180-1

12. Xiao-Jun Chen, Cui-Ping Feng, Cheng-En Mei: Effect of activated ganoderma lucidum and ganoderma spores on the secretion of the splenic cell IL-2 and the transformation and proliferation of the splenic lymphocyte of the rats. Amino Acids and Biotic Resources 2001;23:46-7

13. Qiang Zhang, Gen-Fa Xie, Shu-Xiang Cui: Experiment of ganoderan to increase the immune function of the rats. Shandong Pharmaceutical Industry 1998;17:245.

14. Lin ZB: Focus on anti-oxidative and free radical scavenging activity of Ganoderma lucidium. J Appl Pharmacol 2004;12:133-7

15. Torsten RD, Inga W, Manuela B, Gerhard F, Bernd K: Resistance of p53 knockout cells to doxorubicin is related to reduced formation of DNA strand breaks rather than impaired apoptotic signaling. DNA Repair 2003;2:49-60.
Correspondence to:

Dr. Wei-de Zhong

First Municipal People's Hospital, Guangzhou Medical College, Guangzhou 510180, China

E-mail: wdezhong@21cn.com 